\title{
Inercia y resistencia al cambio en las políticas de drogas: El caso del desarrollo alternativo en Satipo-Perú ${ }^{+}$
}

\author{
MIRYAM NACIMENTO* \\ City University of New York (CUNY)-Graduate Center \\ mnacimentobeltran@gc.cuny.edu
}

https://doi.org/10.18800/rcpg.201601.002

\section{RESUMEN}

Las políticas de desarrollo alternativo (DA) constituyen medidas para el control de la oferta de drogas ilícitas promovidas en países de la región andina con el fin de limitar la producción ilegal de cocaína. Estas políticas han conllevado a la continua creación de una serie de programas y proyectos, los cuales han sido objeto de grandes cuestionamientos dada su limitada efectividad y escaso impacto en la disminución de la oferta internacional de cocaína, así como por sus consecuencias dañinas para las poblaciones locales. A pesar de las críticas, estas políticas han continuado siendo implementadas a lo largo de los años. El artículo busca dar cuenta de esta contradicción en un intento de explicar la inercia o la continua reproducción de intervenciones de desarrollo alternativo en el Perú.

A partir del estudio de un programa de desarrollo alternativo que actualmente está siendo implementado en la provincia de Satipo-Perú, se analiza cómo la inercia es experimentada y perpetuada a nivel local. Utilizando el enfoque de la economía política cultural, el artículo concluye que la inercia es el resultado de la confluencia de intereses particulares y prácticas cotidianas llevadas a cabo por diversos actores, los cuales se anclan en estructuras materiales de desigualdad.

Palabras clave: desarrollo alternativo, inercia, hegemonía, sentido común, coca, Perú.

\footnotetext{
* Magíster en Políticas Públicas del Programa Erasmus Mundus. Licenciada en Ciencia Política de la PUCP. Actualmente cursa estudios de doctorado en el programa de Antropología en el Graduate Center de la City University of New York (CUNY).

+ Recibido el 10 de marzo de 2016; aceptado el 4 de septiembre de 2016.
} 
Inertia and resistance to change in drug policies: The case of alternative development in Satipo-Peru

\begin{abstract}
Alternative Development (AD) policies are initiatives aimed at controlling illicit cocaine supply coming from the Andean region. While Andean states have created a great number of $\mathrm{AD}$ projects and programs, these have been object of harsh criticism given their limited impact on the reduction of global cocaine supply and their harmful consequences for local communities. In spite of criticism, AD policies have been implemented over the last thirty years. This article provides an account of this contradiction by explaining the workings of Alternative Development's inertia or its continuous reproduction in Peru.

By drawing on the case of a particular Alternative Development program that is currently being implemented in the province of Satipo, the article analyzes how policy inertia is experienced and perpetuated at the local level. Using the theoretical approach of Cultural Political Economy, the article concludes that policy inertia is the result of vested particular interests and everyday practices carried out by a wide variety of actors who are anchored in structures of material inequality.
\end{abstract}

Key words: alternative development, inertia, hegemony, common sense, coca, Peru. 


\section{Contexto General}

El régimen de control de drogas constituye el actual arreglo jurídico internacional para controlar la producción, distribución y consumo de drogas a nivel global. En este marco, en el año 1961 la cocaína fue incluida en la lista de sustancias peligrosas y, desde entonces, se sujetó a una serie de medidas prohibicionistas. La hoja de coca es la materia prima para la producción de la cocaína y sus derivados, y aquella es producida principalmente por familias campesinas que viven bajo economías de subsistencia en tres países de la región andina: Colombia, Bolivia y Perú. Una de las políticas promovidas para controlar la oferta de cocaína en estos países es el desarrollo alternativo (DA), ${ }^{1}$ cuyo principal objetivo es el de brindar a los productores de hoja de coca alternativas económicamente viables con el fin de que abandonen sus cultivos ilícitos en el marco de una estrategia de desarrollo integral que tome en cuenta los contextos sociales y culturales (United Nations 2015).

A pesar de que las políticas de DA han sido permanentemente promovidas en la región bajo el paraguas de la cooperación internacional desde la década de 1980, su efectividad (así como la validez del actual régimen de control de drogas) ha sido bastante cuestionada por parte de académicos y diferentes sectores de la sociedad civil. Durante los últimos años la evidencia ha mostrado que este tipo de políticas ha sido poco eficaz en disminuir la oferta internacional de cocaína (United Nations 2013). En este artículo busco explicar la contradicción existente entre, por un lado, la implementación continua de políticas de control de la oferta como el DA y, por otro, la creciente evidencia de sus limitaciones así como las frecuentes demandas por un cambio de enfoque.

En este marco, el objetivo del artículo es entender el funcionamiento de la inercia de las políticas de DA y su resistencia al cambio. Para ello, mi análisis se centra en estudiar cómo esta inercia emerge desde lo local a través de prácticas que permiten la continua producción y reproducción diaria de las políticas de DA. Con este propósito, analizo el caso de un programa de DA que actualmente está siendo implementado en la provincia de Satipo, departamento de Junín, en el Perú. ¿Cómo es que la inercia emerge y se actualiza en prácticas cotidianas que se dan en el marco de una intervención de desarrollo alternativo en Perú? El tema será abordado desde la perspectiva teórica de la economía política cultural.

1 Aunque el DA es una política común en la región andina, existen diferencias en los regímenes de regulación de la producción de hoja de coca entre los países. La situación del cultivo de coca varía en Colombia, Perú y Bolivia. 
Tomando como base la interacción entre ideas, discursos y sus circunstancias materiales en la reproducción de relaciones sociales, haré uso de los conceptos de hegemonía y sentido común, desarrollados por Antonio Gramsci, los cuales me permitirán explicar el fenómeno de la inercia.

El programa de desarrollo alternativo a analizar se denomina Desarrollo Alternativo en Satipo (D.A.S.) y está parcialmente financiado por la Unión Europea. Aunque la cooperación europea —a diferencia de otros entes cooperantes - no condiciona su ayuda a actividades de erradicación de hoja de coca, el D.A.S., como todos los programas de desarrollo alternativo en el Perú, está enfocado en la sustitución de cultivos ilícitos por otros cultivos lícitos como el cacao o el café. Tal y como sucede en otros lugares, el número de beneficiarios del programa es pequeño en comparación con el número total de productores agrarios en la zona, y las actividades que se promueven son la promoción de la producción agraria lícita para la exportación así como el desarrollo de capacidades. Luego de realizar dos salidas de campo logré llevar a cabo un total de veinte entrevistas semiestructuradas. Asimismo, participé en dos talleres dirigidos a la población beneficiaria. De otro lado, analicé también fuentes documentales (reportes y archivos) provistas por consultores del programa y funcionarios de DEVIDA. ${ }^{2}$

El artículo se divide de la siguiente manera. Primero, describiré el escenario general en el que surgen las intervenciones de DA en el Perú dando cuenta también de sus limitaciones. Luego, explicaré la aproximación de la economía política cultural en tanto es el marco teórico que he elegido para entender mi objeto de estudio. A continuación, analizaré la inercia de las políticas de DA a partir del caso de la intervención que viene siendo llevada a cabo en Satipo. Desde una dimensión material, abordaré las relaciones de producción que caracterizan a la industria de la cocaína y al desarrollo alternativo como componente de la política contra las drogas en el Perú. Desde el ámbito ideológico, discutiré los diferentes sentidos comunes que existen en torno al problema de la coca y al desarrollo alternativo, los cuales son creados y moldeados estratégicamente por parte de los diferentes actores que participan en la misma implementación del programa. Todo ello me llevará a reflexionar sobre las dimensiones materiales y discursivas que permiten la continua implementación de las políticas de desarrollo alternativo así como sobre las formas en las que estas son (re)producidas en interacciones cotidianas entre

2 La Comisión Nacional para el Desarrollo y Vida sin Drogas, DEVIDA, es el organismo encargado de conducir la política nacional de lucha contra las drogas en el Perú. 
todos los involucrados. Finalmente, arribaré a conclusiones acerca de las relaciones de dominación y desigualdad que subyacen a las actuales políticas de control de drogas en el Perú.

\section{LAS POLÍTICAS DE DESARROLLO ALTERNATIVO Y SUS LIMITACIONES}

El régimen internacional de control de drogas se encuentra adscrito a las Naciones Unidas. Desde esta organización y a través de la adopción de una serie de tratados, los Estados acordaron limitar la oferta legal de la cocaína a sus usos científicos y médicos, al tiempo que su consumo recreacional y social quedó prohibido. De ahí que las políticas promovidas en países productores de cocaína hayan estado centradas en la supervisión y reducción de los cultivos de hoja de coca, materia prima para la producción de cocaína.

Actualmente existen dos mecanismos para prevenir la desviación de los cultivos de coca a mercados ilegales: la erradicación y los programas de desarrollo alternativo. A pesar de que varios proyectos y programas de DA han existido en la región andina desde de la década de 1980, es a partir de 1998 que el desarrollo alternativo como modelo de política es oficialmente reconocido por las Naciones Unidas. Según la definición de las Naciones Unidas, el desarrollo alternativo consiste en

[...] un proceso destinado a impedir y eliminar el cultivo ilícito de plantas que contengan estupefacientes y sustancias psicotrópicas mediante la adopción de medidas de desarrollo rural expresamente concebidas con tal fin, y que se lleva a cabo en el contexto de un crecimiento económico nacional sostenido y de los esfuerzos por alcanzar un desarrollo sostenible de países que están tomando medidas contra las drogas, teniendo presentes las características socioculturales especiales de las comunidades y grupos destinatarios [...] (UNGASS 1998).

Para el caso de la cocaína, este modelo busca limitar la producción de hoja de coca desviada a mercados ilegales a través de alternativas económicas rentables en el marco de una estrategia de desarrollo rural amplia. En la región andina esto se ha traducido en la creación de una serie de programas y proyectos centrados en la sustitución de cultivos ilícitos por otros supuestamente rentables como el café y el cacao, sin que esto haya implicado la adopción de una estrategia integral de desarrollo rural. ${ }^{3}$

\footnotetext{
3 A pesar de que en teoría el modelo internacional de DA constituye una estrategia amplia que busca abordar las causas estructurales de la producción ilegal de drogas, en la práctica este modelo se ha traducido en políticas nacionales que, con enfoques bastante reduccionistas, se han abocado a la disminución de cultivos ilícitos con poca consideración
} 
En los últimos años, la evidencia ha mostrado que pese a que la erradicación y los programas de desarrollo alternativo han sido implementados por décadas en la región andina, la reducción de cultivos ilícitos ha ocurrido solamente en áreas específicas y sin tener un impacto profundo en la oferta global de cocaína. ${ }^{4}$ En el año 2014, el cultivo global de hoja de coca se incrementó en 10\% respecto a 2013, aunque de acuerdo a las últimas versiones del Informe Mundial de Drogas, este indicador muestra una tendencia a la baja (United Nations 2016). Sin embargo, esto no debería ser tomado como una muestra de éxito, dado que el monto total de producción ilegal de cocaína permanece estable en la región. Esto se debe a que en los últimos años se han logrado avances tecnológicos en la utilización del suelo que han permitido elevar la densidad de los cultivos de coca, provocando también un aumento en su productividad (Torres 2013: 4).

El comportamiento de los indicadores relativos a la disponibilidad de cocaína en países consumidores como los Estados Unidos es también indicativo de este fenómeno. En dicho país el precio estimado de la cocaína ha decrecido mientras que su pureza ha aumentado desde los años noventa, lo cual refleja el fracaso de los esfuerzos orientados a suprimir la oferta de esta sustancia (Werb et al. 2013:. 2). Sumado a esto, existe un consenso en torno a la existencia del "efecto de globo" por el cual la reducción de cultivos en Bolivia o Colombia suele provocar incrementos en Perú, dando como resultado que este sea el segundo productor de hoja de coca del mundo (United Nations 2016). A pesar de la persistencia de estos resultados negativos, los programas de desarrollo alternativo se han venido implementado ininterrumpidamente en Perú con el apoyo de múltiples fuentes cooperantes, lo que revela la existencia de una brecha entre las políticas para el control de drogas y la creciente evidencia de sus limitaciones.

\section{LA INERCIA DESDE LA MIRADA DE LA ECONOMÍA POLÍTICA CULTURAL}

Diferentes perspectivas teóricas han buscado explicar el problema de la inercia, la continuidad y el cambio en la vida social. En particular, vertientes provenientes

\footnotetext{
de los contextos sociales y culturales. Desde la sociedad civil, diferentes sectores han llamado la atención sobre la necesidad de implementar estrategias con un enfoque de desarrollo humano más amplio.

4 Como en el caso de cualquier actividad ilícita, existe una gran dificultad para contar con datos precisos acerca de la producción de cultivos ilícitos. Existen distintas entidades que realizan mediciones anuales sobre esta actividad. Las fuentes principales son el Gobierno de los Estados Unidos y la Oficina de las Naciones Unidas contra el Crimen y las Drogas (UNODC). La falta de criterios y metodologías inequívocas ha provocado disputas y controversias respecto del número oficial de plantaciones de coca en los Andes.
} 
del nuevo institucionalismo ${ }^{5}$ han analizado cómo las estructuras institucionales determinan los fenómenos sociales y políticos. Bajo esta perspectiva, la inercia no es entendida en sí misma como un fenómeno dinámico sino que es sinónimo de «no cambio» (Genschel 1995: 6). Las instituciones son aquí estructuras estables que alteran las expectativas de los individuos y limitan la acción humana. Las instituciones, por lo tanto, constriñen la dinámica política y social (Hay 2011: 67). Visto esto, y a pesar de reconocer la importancia de las instituciones, no pretenderé aquí tomarlas como la base para mi análisis, en tanto que considero que los fenómenos sociales no pueden ser reducidos a formas institucionales.

En su lugar, utilizaré aquí el enfoque de la economía política cultural, la cual constituye una corriente teórica emergente que reintroduce la contribución del giro cultural (la preocupación por la semiosis o la construcción de significado) al análisis de la articulación entre lo económico y lo político en el contexto de las relaciones sociales (Jessop 2010: 336). Para los propósitos de este artículo, esta aproximación supondrá hacer un análisis en dos frentes: primero, de las condiciones materiales que posibilitan la inercia de las políticas de DA en Perú; y segundo, de su activa constitución y reproducción a través de un conjunto de ideas y representaciones en la vida cotidiana de los involucrados. En otras palabras, aquí estudiaré cómo la inercia es actualizada a través de las prácticas políticas que son llevadas a cabo diariamente por los actores. Desde este punto de vista, la inercia no es rígida ni supone necesariamente una oposición al cambio.

Mediante la utilización de una perspectiva neogramsciana podré dar cuenta de ella retomando dos conceptos desarrollados por Gramsci: hegemonía y sentido común. El concepto de hegemonía es utilizado por Gramsci para explicar las formas de dominación y subordinación inherentes a toda práctica social humana (Cox 1983). De acuerdo con él, estas relaciones de dominación tienen lugar en cualquier orden social y dependen simultáneamente del consenso y de la coerción. ${ }^{6}$ La hegemonía es pues «la expresión de un consenso amplio que se manifiesta en la aceptación de ideas y que está sustentado en recursos materiales e instituciones, y todo ello es establecido a través de las fuerzas sociales que ocupan un rol predominante en el Estado» (Bieler \& Morton 2004: 87). Así, la hegemonía se construye prioritariamente a partir de relaciones sociales

\footnotetext{
5 Pese a que el nuevo institucionalismo no es un campo de estudio unificado, existen tres corrientes analíticas que reclaman ser parte de él: el institucionalismo histórico, el institucionalismo de elección racional y el institucionalismo sociológico (Hall y Taylor 1996: 5).

6 En la obra de Gramsci, el concepto de hegemonía es uno de tipo polisémico dado que es usado de diversas maneras en distintos momentos. Para fines de este artículo utilizaremos una interpretación compleja de hegemonía, la cual implica la combinación simultánea de la fuerza material y el consenso.
} 
de producción en formas tanto materiales como discursivas (Cox 1987: I-9). Para fines de este trabajo, el concepto de hegemonía me ayudará a dar cuenta de las relaciones de poder sobre las que se fundamenta la inercia de las políticas de DA; su continua reproducción terminará siendo el resultado de un consenso hegemónico que surge de la interacción entre distintas fuerzas sociales que actúan bajo relaciones de producción específicas.

En tanto la hegemonía implica la construcción de ideas e imaginarios, haré uso también del concepto de sentido común, término utilizado por Gramsci para referirse a los preconceptos que contribuyen (o no) a la reproducción de ciertos regímenes de poder, facilitando u obstruyendo el cambio social (Crehan 2002: 281). De este modo, Gramsci buscó explorar el papel de las ideas en el mantenimiento de la hegemonía. Para él, el sentido común es «la filosofía de los no-filósofos [...] una concepción del mundo que es acríticamente absorbida en los múltiples ambientes sociales y culturales en los que la moral individual del hombre común se desarrolla» (Gramsci 1971: 419). Gramsci asume la existencia de múltiples sentidos comunes debido a la convivencia simultánea de diversos ambientes culturales. Distintos sentidos comunes están socialmente delimitados por sus circunstancias materiales, sin embargo, la manera en la que los sentidos comunes se desarrollan depende también de factores contingentes.

En consecuencia, la hegemonía de un grupo particular (y sus correspondientes sentidos comunes) no es fija ni estable, por lo que necesita ser continuamente creada y recreada en sus prácticas cotidianas. La aceptación o negación de un sentido común hegemónico solo es posible a través de la agencia de los actores que, en su relación con otros actores, promueven que sus propias ideas se vuelvan dominantes. De esta forma, tanto la hegemonía como el sentido común se refieren necesariamente a la existencia de un campo de interacción y lucha entre distintas fuerzas, lo que explica el avance de cualquier tipo de transformación social.

\section{DiNÁMICAS AGRARIAS DE PRODUCCIÓN DE COCA EN SATIPO-JUNÍN}

La continua reproducción de las políticas de DA está condicionada, primero, por las circunstancias materiales que determinan la industria de la cocaína. En este punto es importante recordar que la producción de cocaína constituye, sobre todo, una industria transnacional que se enmarca en relaciones sociales de producción capitalista y, por lo tanto, está inmersa en relaciones de dominación, explotación y de clase. Esto se manifiesta en el hecho de que el Perú solo es parte de los eslabones más bajos y menos lucrativos de esta industria, lo que 
la convierte en una organización con una división de trabajo bien establecida, basada en la mejora de mecanismos tecnológicos en las etapas más elevadas del ciclo de negocio. En la región andina, son peruanos y bolivianos quienes por lo general participan en los estadios inferiores con las actividades de cultivo de hoja de coca y producción de pasta básica de cocaína, mientras que los colombianos suelen estar a cargo del refinamiento, transporte y distribución a los países consumidores (Briones, Cumsille, Henao \& Pardo 2013: 39).

Alrededor del $88 \%$ de la producción de coca en el Perú se concentra en tres zonas: el valle del Alto Huallaga, el valle del río Apurímac, Ene y Mantaro (VRAEM), y la Convención-Lares (Unión Europea \& República del Perú 2010). El VRAEM es una de las zonas más importantes de cultivo de hoja de coca, en tanto es responsable de más del $50 \%$ de la producción en todo el país (Unión Europea \& República del Perú 2010). La producción restante proviene de regiones fronterizas con Colombia, Bolivia y la Selva Central. Satipo es una provincia ubicada en la región de Junín, parte de la Selva Central. La mayoría de su población está compuesta por migrantes andinos que viven en áreas rurales en condiciones de pobreza. Dada su proximidad con el VRAEM, Satipo es considerada una puerta de acceso a este valle.

El mercado de la hoja de coca en Satipo tiene características particulares que derivan de su simultánea participación en dinámicas económicas locales, nacionales e internacionales (Goodhand, Meehan \& Pérez-Niño 2014). La producción ilegal de coca en Satipo es producto de la dispersión espacial de los cultivos provocada por dinámicas nacionales del llamado «efecto globo». Una gran cantidad de campesinos andinos migraron a Satipo el año 2000 luego de huir de una serie de iniciativas de erradicación forzosa que fueron implementadas en la región del Alto Huallaga durante ese periodo (van Dun 2009: 95). Asimismo, la economía de Satipo está prioritariamente dominada por el café y el cultivo de coca. Dadas sus condiciones materiales (nivel de adaptabilidad a diferentes tipos de suelo, mayor frecuencia de la cosecha y bajos costos de producción), así como por su familiaridad histórica con la población local, la hoja de coca es el cultivo que más se adapta a una economía rural de bajos recursos, con poco acceso a capital y a tecnologías, como la de Satipo. Como Buxton sugiere, ningún otro cultivo brinda la misma cantidad de beneficios en condiciones de marginalidad (Buxton 2015: 27). En otras palabras, hay una correspondencia entre los modos de producción agrícola en la Amazonía peruana y el cultivo de hoja de coca. En cuanto a la producción de cocaína, esta se lleva a cabo propiamente en el VRAEM. Satipo es considerada una zona de amortiguamiento, por lo que el 
programa de DA en esta provincia fue creado con el fin de evitar la expansión de actividades ilegales a más zonas.

\section{El programa de desarrollo alternativo en Satipo}

De otro lado, la continua reproducción de la política de DA está condicionada también por cómo se organizan estas intervenciones en tanto componentes primordiales de las políticas contra las drogas en el Perú. Como en el caso de la industria de la cocaína, el componente del DA tiene también una clara división del trabajo que opera a distintas escalas y niveles, tanto local, nacional y global. A pesar de que la burocracia estatal coordina las intervenciones de DA, los actores participantes no son solo entidades estatales, sino que son también entidades cooperantes, consultores y organizaciones no gubernamentales. Todos ellos forman parte de las intervenciones de DA, con intereses propios y expertises particulares. Cabe resaltar el rol central que cumple la cooperación internacional y, por tanto, la importancia de la relación existente entre países donantes y receptores. Esto se expresa en el hecho de que en el Perú los programas de DA fueron originalmente una iniciativa de la cooperación internacional. ${ }^{7}$ Para Tomohisa Hattori (2001) la cooperación internacional consiste en una forma de asignación de recursos voluntaria que no involucra ningún tipo de retorno o de obligación de pago posterior, por lo que es percibida como un regalo unidireccional que un país le hace a otro (Hattori 2001: 636). La relación social que aquí se establece se basa en la existencia de una desigualdad material entre donantes y beneficiarios (uno tiene recursos y el otro no), lo que crea una jerarquía social (Hattori 2001: 636). Como veremos, en el caso del D.A.S., esta relación asimétrica está presente en las diferentes fases y actividades del programa.

El programa de DA en Satipo fue creado por medio de la firma de un convenio de financiamiento entre el Gobierno peruano y la Unión Europea. Este convenio fue establecido a un alto nivel diplomático entre representantes de la Agencia de Cooperación Internacional (APCI) y la Comisión Europea. El documento estipula que el programa debe ser implementado dentro de un periodo de 72 meses con un presupuesto total de $€ 10^{\prime} 400,000$, de los cuales $€$ 8’000,000 provienen de la Unión Europea y € 2’400,000 del Gobierno peruano. Asimismo, la Unión Europea supervisa el cumplimiento de las actividades del programa por medio del monitoreo de su ejecución financiera. En el acuerdo

\footnotetext{
Antes del ańo 2007, los recursos financieros de los programas de DA provenían íntegramente de donantes (siendo USAID y las Naciones Unidas los principales). Es solo a partir del ańo 2007 en adelante que el Gobierno peruano decide asignar sus propios recursos para las intervenciones de DA.
} 
también se determina que la implementación del programa debe seguir ciertos parámetros predeterminados por la Unión Europea, los cuales están recogidos en un protocolo que debe ser seguido por parte del Estado peruano durante toda la gestión del presupuesto-programa. Este protocolo constituye, así, un vehículo por medio del cual, desde el plano internacional, se introducen una serie de valores y modelos de comportamiento a seguir a escala local, lo que termina reproduciendo una relación desigual entre donantes y receptores.

Al nivel nacional, el programa de DA en Satipo se gestiona de forma parcialmente descentralizada. DEVIDA es la entidad responsable de la administración financiera y técnica. La relación entre las oficinas centrales de DEVIDA ubicadas en la capital del país y el personal local del programa que trabaja en Satipo está centrada en el cumplimiento de metas estipuladas en el presupuesto, lo que terminará siendo el único criterio para determinar la eficiencia y eficacia del programa. Esto se expresa en las constantes solicitudes y requerimientos que se le hacen al personal del D.A.S. para dar cuenta del cumplimiento de metas financieras preestablecidas. La lógica de la gestión por resultados permea así toda la implementación, en tanto es necesario probar en todo momento la existencia de una clara articulación entre los productos dirigidos a las poblaciones objetivo y los resultados obtenidos, los cuales deben ser tangibles y ubicables en matrices lógicas a través de indicadores de verificación claros.

En suma, la reproducción o inercia de las políticas de DA en el Perú estará condicionada también por cómo se organizan y coordinan estas intervenciones. Por un lado, las relaciones entre entidades de la cooperación internacional, el Estado peruano, el Gobierno central, los Gobiernos locales y los beneficiarios son de tipo asimétrico y, como tal, todos ellos tienen una capacidad de influencia diferenciada en la formulación y el accionar del programa. Al mismo tiempo, este tipo de relacionamiento asimétrico se da en el contexto de la gestión por resultados, siendo las herramientas de gestión los elementos centrales por medio de los cuales se lleva a cabo la implementación de las intervenciones.

\section{EL FRACASO COMO SENTIDO COMÚN EN LAS INTERVENCIONES DE DA}

Como habíamos mencionado, la noción de sentido común desarrollada por Gramsci se refiere a las distintas concepciones del mundo que guían la acción social. Existen en el marco de las políticas de DA distintos sentidos comunes referidos al problema de los cultivos ilícitos, los cuales se interrelacionan entre sí al ser negociados y defendidos por distintos actores. Aquí veremos cómo es que a partir de las interacciones que se dan en el marco del D.A.S., algunos 
sentidos comunes reciben mayor aceptación que otros. Así, me enfocaré en estudiar aquellos sentidos comunes que se refieren a la producción de dos ideas que considero fundamentales: la idea de «éxito» y la idea de "fracaso» de las intervenciones de desarrollo alternativo. La construcción de estas dos ideas es de particular importancia dado que es sobre la base de ellas que la inercia de las políticas de drogas se perpetúa en la práctica.

La mayoría de actores con los que conversé durante mis visitas de campo dio cuenta de las grandes limitaciones y problemas del DA. En efecto, el fracaso es un sentido común bastante generalizado e internalizado por los actores, quienes lo reconocen desde distintas posiciones. Una de las razones evocadas continuamente por los funcionarios del programa es la ausencia de una estrategia nacional e integral de DA (a pesar de la existencia de intervenciones que cumplen sus propósitos de manera aislada). El escaso dinero asignado por el Estado al DA, así como el poco personal que se encuentra trabajando en el campo y en condiciones precarias, habría provocado que los diferentes programas formulados terminen siendo intervenciones de baja intensidad capaces de abarcar solo unos pocos distritos dentro de su ámbito de acción, lo que habría limitado todo su impacto.

De otra parte, para los campesinos beneficiarios del D.A.S. la historia es un poco diferente. Aunque estos también suelen reconocer las grandes limitaciones en las intervenciones de DA, para muchos de ellos la causa del fracaso no es la falta de estrategias integrales, sino que la coca sería un cultivo imposible de ser erradicado de los suelos peruanos. Incluso en caso de existir mejores estrategias, para ellos, la coca siempre será parte de la agricultura peruana. En la opinión de un agricultor participante del programa: «a pesar de todo, los campesinos siempre van a producir coca y los traficantes también van a hacer su parte. Aquí la coca aquí crece sola... ¡es parte de nuestra cultura!» (comunicación personal, 15 de junio de 2014). Asimismo, el mismo agricultor expresó que el Gobierno debería contemplar la legalización de la cocaína, dado que los programas de DA no brindan opciones viables para dejar de producir coca: «Si el Gobierno no quiere que la producción se vaya al tráfico de drogas, entonces este debería comprar la coca de los campesinos y luego tirarla al mar. Esta sería una mejor alternativa que el desarrollo alternativo» (comunicación personal, 15 de junio de 2014).

Por su parte, algunos consultores del programa — quienes participan solo temporalmente en algunas actividades del mismo- manifestaron también posturas bastante críticas respecto de las intervenciones de DA en general. Por 
un lado, algunos hicieron alusión a la debilidad institucional de DEVIDA. Un consultor del programa, por ejemplo, manifestó:

DEVIDA no tiene ningún poder real para reunir a todos los actores involucrados en el problema de las drogas. Esta institución no tiene la llegada suficiente para coordinar con otros sectores y cambiar las políticas agrarias o las políticas comerciales que afectan a los campesinos cocaleros (comunicación personal, 25 de junio de 2014).

Asimismo, la misma conceptualización de DA utilizada en los programas fue también duramente criticada por diversos consultores al ser calificada de obsoleta, errada e incluso perniciosa. En palabras de un consultor, el DA tal como se entiende hoy en día "condena a los campesinos a la pobreza» (comunicación personal, 25 de junio de 2014). En su opinión, el error está en el énfasis que se le da al enfoque agrario: «el DA ha sido interpretado aquí como una alternativa rural a la coca... pero ¿por qué necesariamente tiene que ser una alternativa rural? ¡Este es el primer error! El DA no debería estar limitado al sector rural» (comunicación personal, 25 de junio de 2014). Para él, las intervenciones de DA deberían promover actividades que se lleven a cabo fuera de los ámbitos rurales y existirían diversas razones para ello. Primero, la rentabilidad de los cultivos alternativos como el café y el cacao suele ser bastante baja, a lo que se añade las grandes restricciones que los campesinos deben enfrentar para colocar sus productos alternativos en los mercados internacionales. Ni el Gobierno ni las intervenciones de DA pueden cambiar la desventaja estructural que tienen los pequeños campesinos en el ámbito internacional. De otra parte, el acceso a la propiedad de la tierra es también bastante restringido para ellos, dado que el Estado no ha logrado aún expandir la tenencia de tierra a pequeños agricultores. Estos, entre otros, fueron algunos de los motivos que diferentes consultores del programa mencionaron respecto a la incapacidad del DA para convertir a los campesinos cultivadores de coca en exitosos emprendedores rurales.

Algunos representantes de organizaciones indígenas beneficiarias del programa ofrecieron también una visión similar acerca de los programas de DA. Ellos expresaron que en el campo la gente sabe que ningún programa de DA puede ofrecer ninguna solución a la producción ilegal de drogas. Así, alguna vez un representante indígena me comentó claramente su posición:

Yo creo que incluso DEVIDA reconoce que ninguna de sus intervenciones es de alto impacto. Ellos están bastante conscientes de eso. Y nosotros también, por eso a veces nos frustramos (representante indígena, comunicación personal, 20 de junio de 2014). 
La mayoría de entrevistados relataron experiencias semejantes. Todos expresaron que no existe una estrategia de desarrollo alternativo comprehensiva que sea capaz de responder a las necesidades de generación de ingresos de los pequeños campesinos. A pesar ser este el objetivo oficial del modelo de DA tal y como fue ideado por las Naciones Unidas, en la práctica ninguno de los programas implementados está ni siquiera cerca de alcanzarlo.

Lo claro es, entonces, que la mayoría de actores está de acuerdo con que los programas de DA no están alterando las causas estructurales de la producción ilegal de coca. Esta es, en suma, la narrativa que sustenta el sentido común del fracaso de las intervenciones de DA, la cual suele ir acompañada de la serie de motivos ya mencionados, tales como el reconocimiento de la vulnerabilidad de pequeños campesinos, la reivindicación cultural del uso y producción de la coca, así como del cuestionamiento a la sustitución de plantaciones ilegales en contextos rurales.

\section{LA PRODUCCIÓN PERMANENTE DEL «ÉXITO» Y LA PERPETUACIÓN DEL MODELO DE DA}

Visto esto, las preguntas que es preciso hacerse son: ¿’or qué las intervenciones de DA siguen siendo implementadas si el fracaso es un sentido común tan generalizado y aceptado abiertamente? ¿Por qué los diferentes actores consienten participar activamente en este tipo de programas después de todo? Es claro que la implementación de cualquier iniciativa requiere de la conformidad y cierto nivel de compromiso de los que participan en ella. Mi respuesta ante esta aparente contradicción es que esto sucede debido a que el sentido común del «éxito» del DA está continuamente obteniendo una posición hegemónica independientemente de las críticas y de los hechos. Y es en la práctica de la implementación del programa y a través de la negociación entre las partes que esta idea del éxito del DA obtiene una aceptación oficial. ¿En qué consiste esta idea del éxito que sustentaría la reproducción continua de programas de DA? Y, sobre todo, ¿cuáles son esas prácticas que la apoyan?

A pesar de reconocer la falta de una política comprehensiva e integral capaz de crear las condiciones necesarias para que los campesinos abandonen sus cultivos ilícitos, los funcionarios del programa de DA en Satipo manifestaron en varios encuentros que este era un programa exitoso, sustentándose en el hecho de que las actividades programadas estaban siendo implementadas de acuerdo a los requerimientos estipulados. En otras palabras, el éxito aquí está referido primordialmente a la consecución de metas operativas y a la obligación 
de ejecutar el presupuesto. Los programas de DA considerados exitosos, en realidad, no son exitosos debido a que dirijan sus esfuerzos a alterar las condiciones estructurales de la producción de coca (como en teoría el modelo de DA sugiere). Más bien, el éxito parece estar aquí desligado del propósito original del modelo de la política, y consiste esencialmente en cumplir con lo estipulado en el marco lógico del programa.

El éxito se convierte así en un sentido común hegemónico a través de prácticas de gestión cotidianas llevadas a cabo por funcionarios y consultores, quienes deben dar cuenta de la consecución de resultados preestablecidos. Los resultados son centrales en el diseño del programa y son constantemente evocados en su implementación diaria mediante la utilización de datos e indicadores incorporados a las matrices lógicas y otras herramientas de planificación. Es así que, a través del cumplimiento de metas establecidas en estas herramientas de planificación y gestión, se demuestra el éxito del programa.

$\mathrm{Al}$ mismo tiempo, en un segundo movimiento, esto implica que el programa adquiere también control sobre la interpretación del problema de los cultivos ilícitos y el DA. En otras palabras, se da también la estabilización de una interpretación hegemónica sobre el DA, la cual es producida y reproducida socialmente a través de prácticas de gestión a nivel local (Mosse 2004: 646). Las herramientas de gestión cumplen por ello también la función de traducir la abstracción del modelo internacional de desarrollo alternativo a la práctica local, instaurándose así una versión particular de este modelo en la implementación del programa, la cual termina siendo la oficial. De esta forma, el modelo de DA, alguna vez discutido en las Naciones Unidas y acordado a nivel diplomático entre los Estados, se crea (y se recrea) como un campo de intervención a través las prácticas cotidianas de gestión llevadas a cabo por diferentes funcionarios y consultores a nivel local.

Un ejemplo de cómo la política de DA se produce y reproduce preservando representaciones hegemónicas es el claro enfoque de mercado que está implícito en algunas actividades del programa. En estas se estipula que las municipalidades dentro de su jurisdicción reciban asistencia técnica para mejorar el registro de tierras con el fin ulterior de su titulación. El objetivo es convertir a los campesinos en emprendedores agrícolas, partiendo de la premisa de la eficiencia de los mercados. Desde esta perspectiva, los títulos de propiedad serían capaces de brindar desarrollo económico, dado que estos asegurarían los derechos de propiedad así como la acumulación de capital mediante la reducción de los costos de transacción. Gracias a los títulos de propiedad, los 
campesinos podrían acceder a crédito con la finalidad de realizar inversiones que conllevarían a la capitalización de su agricultura. Como consecuencia final, la productividad de los cultivos lícitos incrementaría y los campesinos abandonarían las plantaciones ilegales de coca.

Sin embargo, este sentido común implícito en las actividades del programa asume la existencia de la eficiencia de mercados sin tomar en cuenta la desigualdad económica característica de la organización de la producción agraria a nivel internacional, nacional y local. Los mercados agrarios están lejos de ser espacios neutrales en donde los actores puedan competir en igualdad de condiciones. En realidad, los altos niveles de pobreza así como el acceso limitado a servicios socaban la competitividad de los pequeños productores agrarios en el Perú. Al no reconocer esta situación, los programas de DA funcionan como si estuviesen excluidos de las relaciones de poder en las que se inscribe el problema del cultivo ilícito de coca. Las grandes desigualdades presentes en el sistema de comercio internacional, como el limitado acceso a los mercados internacionales por parte de los pequeńos productores agrarios del Sur global, no son tomadas en cuenta. En su lugar, las intervenciones de DA están centradas en capitalizar a los campesinos pobres en regiones productoras de coca, como si estos se encontraran fuera de estas estructuras sociales. De esta forma, las actividades de titulación de tierras suponen una manera particular de definir el problema de la producción ilegal de coca que evita confrontar sus causas estructurales en contextos rurales. Es a través de herramientas de planificación y prácticas cotidianas de gestión que este tipo de sentidos comunes se vuelven hegemónicos, crean la idea del «éxito» y terminan (re)produciendo el modelo de DA a nivel local.

Es importante subrayar también que la hegemonía de estos sentidos comunes está basada sobre las condiciones materiales previamente descritas. Como hemos visto, los diferentes actores involucrados en las políticas de DA están insertos en relaciones sociales que dan cuenta de una desigualdad material. Esto implica que los actores tienen un acceso diferenciado a la formulación de los objetivos de los programas de DA, así como a la manipulación de herramientas de gestión $y$, por lo tanto, sus interpretaciones particulares acerca del problema de la coca tienen también diferentes posibilidades de ser preservadas y mantenidas como hegemónicas. Como vimos, las actividades de codificación y planificación que se llevan a cabo en el marco del programa se corresponden con demandas externas (como en el caso del protocolo establecido por la Unión Europea), a través de las que asumen las perspectivas e intereses de la cooperación internacional, la 
cual tiene mucha más capacidad para influenciar la formulación de este tipo de proyectos que los mismos campesinos beneficiarios.

\section{LA FUNCIONALIDAD DEL «ÉXITO»}

Cabe señalar, al mismo tiempo, que el éxito del programa es aceptado como un sentido común hegemónico y oficial debido a que es también funcional a los intereses de todos los involucrados. En otras palabras, los intereses materiales y deseos personales de campesinos, funcionarios y consultores están estratégicamente vinculados a las actividades del programa. En diferentes ocasiones, diversos actores me expresaron su pragmatismo respecto al motivo de su participación en el D.A.S., dejando claro que es gracias a este programa que todos los involucrados reciben una serie de recursos y beneficios que terminan siendo utilizados estratégicamente para llevar a cabo sus intereses particulares y promover sus agendas. Por ello, a pesar de que exista poca satisfacción con este tipo de intervenciones, los actores piensan que igual es útil participar en ellas.

Esta funcionalidad es muy clara en el caso de los campesinos. Como parte del proyecto, ellos reciben semillas, fertilizantes, así como el análisis gratuito de sus suelos. En la mayoría de casos, los campesinos están convencidos de que la intervención no logrará reducir sus plantaciones ilegales, sin embargo, participan en las actividades porque les es útil para su estrategia de diversificación productiva. Para asegurarse mayores ingresos económicos, los campesinos manejan diferentes cultivos al mismo tiempo, al punto que muchas veces las plantaciones ilegales de coca se encuentran junto a aquellas plantaciones promovidas en el marco del programa. En palabras de un campesino entrevistado: «uno necesita tener cuatro cańos abiertos al mismo tiempo. Uno no puede depender enteramente solo de un cultivo... y menos del café porque no cubre los costos de inversión» (comunicación personal, 10 de julio de 2014). A pesar de que las asociaciones de productores que participan en el D.A.S. han firmado un acuerdo en el cual se comprometen a no tener cultivos ilegales, más allá de este acuerdo, el programa no dispone de ningún mecanismo para confirmar si es que esto ocurre realmente.

De otra parte, los funcionarios que trabajan para el D.A.S. también se convierten en defensores activos del programa, dado que este también es funcional a sus intereses de desarrollo profesional y reputación. Como me expresó alguna vez un consultor del programa:

[...] los que trabajan en DA quieren asegurar sus puestos. Viven de eso. Hay gente que sí trabaja con cierta ética profesional y quieren gastar el dinero de forma eficiente, pero ellos se preocupan principalmente por sus salarios y privilegios, entonces no es que 
puedan ir en contra de la cooperación y los proyectos (comunicación personal, 10 de julio de 2014).

De esta forma, distintos actores se relacionan entre sí y consienten su participación en los programas de DA, sacándole el mayor provecho a su involucramiento, a pesar de que sus posturas particulares respecto al problema de la coca en muchos casos se opongan y hasta subviertan la narrativa oficial del D.A.S. Tanto implementadores como beneficiarios aprovechan estratégicamente los recursos del programa al tiempo que desarrollan acciones acordes a los cánones oficiales a fin de producir evidencias de su aparente éxito, pese a que las partes mantienen una posición abiertamente cínica sobre la plausibilidad de los objetivos oficiales del DA. Este escenario produce una curiosa forma de hegemonía que no se basa en la naturalización de ciertos sentidos comunes acerca de la pertinencia de los medios y fines del DA, sino en un consenso tácito por el cual el DA es un medio útil para alcanzar los intereses particulares de todas las partes. La inercia del DA es así (re)producida desde lo local gracias a la confluencia de todas estas posiciones e intereses convergentes.

\section{Conclusiones}

Mediante el análisis de las condiciones materiales, las prácticas de gestión y los sentidos comunes existentes en la implementación del D.A.S., he ilustrado en este artículo los diferentes factores que hacen posible la inercia o reproducción continua de las intervenciones de desarrollo alternativo a pesar de su inefectividad. Así, vimos cómo las relaciones de producción actuales ubican a los productores de hoja de coca en Satipo en los eslabones más bajos de la industria de la cocaína y de la organización del DA. Esta asimetría material constituye la base sobre la que se asienta la continuidad del DA en tanto política ineficaz. Asimismo, analizamos cómo diversos sentidos comunes opuestos y contradictorios entre sí conviven en la implementación del programa analizado. Por un lado, dimos cuenta de la idea generalizada del fracaso de este tipo de intervenciones debido a que su impacto en la reducción de cultivos es limitado y cuestionable. De otro lado, vimos también cómo las intervenciones de DA siguen funcionando y reciben un apoyo constante debido a que el éxito de los programas se convierte en un sentido común hegemónico a través de la aceptación de indicadores y consecución de metas operativas, las cuales llevan implícitas interpretaciones particulares y parcializadas acerca del problema de la coca en el Perú y que no confrontan sus causas estructurales. De esta forma, los objetivos e indicadores del programa terminan estando descontextualizados de las dinámicas agrarias y 
económicas que hacen que los campesinos produzcan coca de manera ilegal, al tiempo que estos indicadores circulan cotidianamente en los diferentes espacios generados por el programa, legitimándose así el modelo de DA ante el Estado y la cooperación internacional.

Finalmente, el programa de DA en Satipo se reproduce también debido a que este termina siendo compatible con los intereses y agendas de todos los involucrados. Por un lado, los recursos que el programa moviliza (fertilizantes, semillas, legitimidad, entre otros) hacen posible satisfacer los requerimientos formales de la cooperación internacional sobre el DA; por otro lado, estos también son utilizados estratégicamente por parte de campesinos, funcionarios y consultores para satisfacer sus intereses inmediatos. La inercia de las políticas de DA termina siendo así aquella situación hegemónica que resulta de la validación cotidiana y la convergencia de intereses, a pesar de que todos los actores involucrados reconozcan sus limitaciones e incluso su fracaso.

\section{BibLIOGRAFÍA}

Bieler, A. \& A. D. Morton (2004). «A critical theory route to hegemony, world order and historical change: neo-Gramscian perspectives in International Relations». Capital \& Class. No. 28(82), pp. 85-114.

https:/doi.org/10.1177/030981680408200106

Briones, Á.; F. Cumsille; A. Henao \& B. Pardo (eds.) (2013). The Drug Problem in the Americas. Analytical Report. Organization of American States, General Secretariat.

Buxton, J. (2015). Drugs and Development: the Great Disconnect. Global Drug Policy Observatory, Swansea University. Policy Report 2, january.

https:/doi.org/10.1177/03058298830120020701

Cox, R. W. (1983). «Gramsci, Hegemony and International Relations: An Essay in Method». Millennium-Journal of International Studies. No. 12(2), pp. 162-175.

https:/doi.org/10.1177/03058298830120020701

Cox, R. W. (1987). Production, power, and world order: social forces in the making of history. New York: Columbia University Press.

Crehan, K. A. F. (2002). Gramsci, culture and anthropology. Berkeley: University of California Press.

Genschel, P. (1995). "The dynamics of inertia: institutional persistence and change in telecommunications and health care». MPIFG Discussion Paper. No. 95/3, 10(1), pp. 1-32.

Goodhand, J., P. Meehan \& H. Pérez-Niño (2014). «Drugs, (dis)order and agrarian change: the political economy of drugs and its relevance to international drug policy». Occasional publication. Norwegian Peacebuilding Resource Centre, october.

Gramsci, A. (1971). Selections from the prison notebooks of Antonio Gramsci [electronic resource]. New York: International Publishers.

Hall, P., \& Taylor, R. (1996). Political science and the three new institutionalisms. Political Studies, 44(5), 936-957.

https:/doi.org/10.1111/j.1467-9248.1996.tb00343.x 
Hattori, T. (2001). «Reconceptualizing Foreign Aid». Review of International Political Economy. No. (4), 633. https:/doi.org/10.1080/09692290110077610

Hay, C. (2011). «Ideas and the Construction of Interests». En Béland, D. \& R. H. Cox (Eds.), Ideas and politics in social science research. Oxford, New York: Oxford University Press, pp. 65-82. https:/doi.org/10.1080/19460171003619741

Jessop, B. (2010). «Cultural political economy and critical policy studies». Critical Policy Studies. No. 3(3-4), pp. 336-356. https:/doi.org/10.1080/19460171003619741

Mosse, D. (2004). «Is Good Policy Unimplementable? Reflections on the Ethnography of Aid Policy and Practice». Development \& Change. No. 35(4), 639-671. http://doi.org/10.1111/j.0012-155X.2004.00374.x

Torres, J. M. (2013). Comentarios al informe 'El Problema de las Drogas en las Américas- 2013' de la Organización de Estados Americanos. Centro de Investigación Drogas y Derechos Humanos.

Ungass (1998). Measures to enhance international cooperation to counter the world drug problem. General Assembly UNGASS S/20/4.

Unión Europea y República del Perú (2010). Convenio de Financiación DCI-ALA/2010/022-032.

United Nations (2013). World Drug Report, 2013. United Nations.

United Nations (2015). Our work [United Nations Office on Drugs and Crime]. Recuperado a partir de http://www.unodc.org/unodc/en/alternative-development/our-work.html

United Nations (2016). World Drug Report 2016. United Nations.

https://www.unodc.org/doc/wdr2016/WORLD_DRUG_REPORT_2016_web.pdf

Van Dun, M. (2009). Cocaleros. Violence, Drugs and Social Mobilization in the Post-Conflict Upper Huallaga Valley, Peru. Utrecht: Rozenberg Publishers. Recuperado a partir de http://dspace. library.uu.nl/handle/1874/33733

Werb, D., T. Kerr, B. Nosyk, S. Strathdee, J. Montaner \& E. Wood (2013). «The temporal relationship between drug supply indicators: an audit of international government surveillance systems». BMJ Open. No. 3(9), e003077-e003077.

http://doi.org/10.1136/bmjopen-2013-00307 